\title{
Equivalence Checking of Hierarchical Combinational Circuits
}

\author{
Williams, Poul Frederick; Hulgaard, Henrik; Andersen, Henrik Reif
}

Published in:

Proceedings of the 6th IEEE International Conference on Electronics, Circuits and Systems

Link to article, DOI:

10.1109/ICECS.1999.812296

Publication date:

1999

Document Version

Publisher's PDF, also known as Version of record

Link back to DTU Orbit

Citation (APA):

Williams, P. F., Hulgaard, H., \& Andersen, H. R. (1999). Equivalence Checking of Hierarchical Combinational Circuits. In Proceedings of the 6th IEEE International Conference on Electronics, Circuits and Systems (pp. 355360). IEEE Press. https://doi.org/10.1109/ICECS.1999.812296

\section{General rights}

Copyright and moral rights for the publications made accessible in the public portal are retained by the authors and/or other copyright owners and it is a condition of accessing publications that users recognise and abide by the legal requirements associated with these rights.

- Users may download and print one copy of any publication from the public portal for the purpose of private study or research.

- You may not further distribute the material or use it for any profit-making activity or commercial gain

- You may freely distribute the URL identifying the publication in the public portal 


\title{
Equivalence Checking of Hierarchical Combinational Circuits
}

\author{
Poul Frederick Williams Henrik Hulgaard Henrik Reif Andersen \\ Department of Information Technology, Building 344 \\ Technical University of Denmark, DK-2800 Lyngby, Denmark \\ E-mail: $\{$ pfw, henrik, hra\}@it.dtu.dk
}

\begin{abstract}
This paper presents a method for verifying that two hierarchical combinational circuits implement the same Boolean functions. The key new feature of the method is its ability to exploit the modularity of the circuits to reuse results obtained from one part of the circuits in other parts. We demonstrate the method on large adder and multiplier circuits.
\end{abstract}

\section{INTRODUCTION}

Due to the increase in the complexity of design automation tools and the circuits they manipulate, such tools cannot in general be assumed to be correct. Instead of attempting to formally verify the design automation tools, a more practical approach is to formally check that a circuit generated by a design automation tool functionally corresponds to the original input. This paper presents a technique for formally verifying that two hierarchical combinational circuits implement the same Boolean functions. The presented technique can also be used to check manual modifications of a circuit to ensure that the designer has not introduced errors. Furthermore, the technique can be used to solve sub-problems of other (higher-level) verification problems. For example, verifying arithmetic circuits by checking that they satisfy a given recurrence equation [6] or verifying the equivalence of two state machines without performing a state traversal [16].

In this paper we use a hierarchical model of combinational circuits. Based on this model, we show how to propagate a cut through two circuits from the inputs to the outputs. The key new feature of the method is its ability to reuse previously calculated results in the verification. Consider the 4-bit adder in Figure 1. The description consists of two cells; a full-adder cell, $\mathrm{fa}$, and a 4-bit adder cell, 4bitadder, containing four instantiations of the full-adder cell and a description of how they are interconnected. The traditional way of verifying hierarchical combinational circuits is to flatten them into

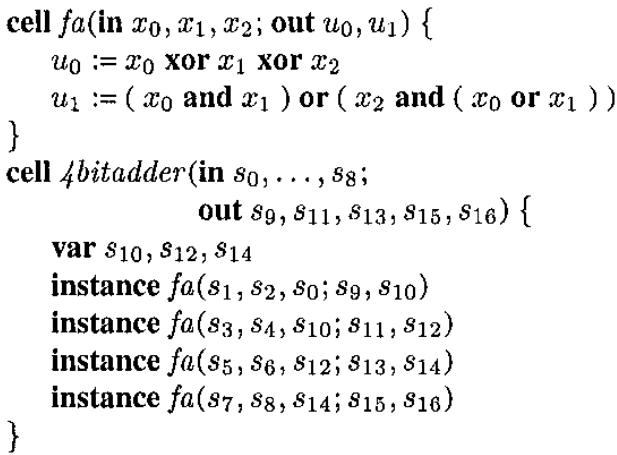

Figure 1: A 4-bit adder consisting of a full-adder cell and a 4-bit adder cell with four instantiations of the full-adder cell.

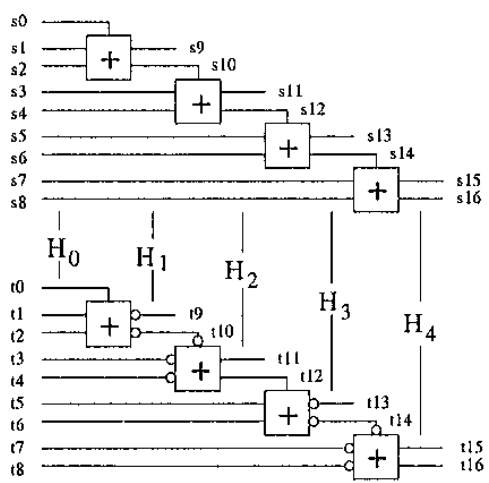

Figure 2: Two 4-bit adders.

a single block of combinational logic on which the verification is performed. In case of complex circuits, this method is not feasible. Our method attempts to work on one cell, and then reuse information about this cell whenever possible.

The 4-bit adder circuit described above corresponds to the top circuit in Figure 2. The bottom circuit in the figure is also a 4-bit adder, but with two instantiations of two different full-adder cells which negate either the inputs or the outputs.

Our method compares the full-adder from the top circuit with each of the two different full-adders in the bottom circuit and combines the results to prove that the two 
circuits are indeed identical (except for some negated inputs and outputs). The method is automatic as it requires no human interaction during the verification process. If the adders in Figure 2 were larger, our method would still only considers two comparisons between fulladders. The rest of the verification would reuse the comparisons to prove the equivalence.

\subsection{Related Work}

Ordered Binary Decision Diagrams (OBDDs) [3] is a data structure for representing Boolean functions. A traditional way of verifying two combinational circuits to be equivalent is to build the OBDDs for the circuits and check if the outputs have identical representations. The OBDDs represent the functionality of the circuits, and some functions have no succinct OBDD representation. Therefore it is not always possible to use standard OBDD techniques. Other canonical decision diagrams have been proposed for verification of combinational circuits. One can use other types of decomposition rules [11], relax the variable ordering restriction $[7,9]$, or extend the domains and/or codomains to integers instead of Booleans [4]. These extensions are typically targeted to solving a particular class of problems.

We have studied the combinational logic-level verification problem for flat circuits [8] using a graph data structure called Boolean Expression Diagram (BED) [1]. This approach works well if the two circuits are similar in structure. However, if the two circuits are very dissimilar in structure, the BED method has the same performance as OBDD methods.

Cerny et al. [5] split circuits into cells and each cell is described by a relation between the inputs and the outputs of the cell. Using a sweep strategy, they move either forwards or backwards through the circuits calculating the relations between the circuits along a cut.

Another approach is a structural method which exploits similarities between the two circuits that are compared by identifying related nodes in the circuits and using this information to simplify the verification problem $[2,12]$. Such techniques rely on the observation that if two circuits are structurally similar, they will have a large number of internal nodes that are functionally equivalent. Eijk and Janssen [17] use the canonicity of OBDDs to determined whether one node is functionally equivalent to another.

Kunz et al. [13] use recursive learning techniques for finding logical implications between nodes in two circuits. Combining learning with OBDDs leads to techniques which can verify larger circuits [15]. The learning technique is further extended by Jain et al. [10] and by Matsunaga [14], introducing more general learning methods based on OBDDs and better heuristics for finding cuts in the circuits to split the verification problem into more manageable sizes.

The main differences between all the methods above and our proposed method is that they use a flat circuit de- scription while we use a hicrarchical one, and they cannot reuse previously calculated results.

\section{HiERARCHICAL COMBINATIONAL Circuits}

Most circuit description languages, for example Berkeley Logic Interchange Format (BLIF), contain language constructs for modular circuit descriptions. Modules may contain other modules yielding a hierarchical description. This leads to a model of hierarchical combinational circuits based on cells, instantiations of cells, and connecting wires. There are two types of cells: those that contain instantiations of other cells, container cells, and those that contain logic gates, logic cells. Definition 1 , 2 , and 3 define a mathematical model for hierarchical combinational circuits based on these observations.

Definition 1 (HCC) A hierarchical combinational circuit $(H C C)$ is a pair $(C, c)$, where $C$ is a set of cells and $c \in C$ is the top cell.

For example, Figure 1 describes an $\operatorname{HCC}(C, c)$, where $C=\{f a, 4$ bitadder $\}$ and $c=4$ bitadder

Definition 2 (Cell) A cell $c$ has the following attributes:

Vars (c), a set of variables,

In $(c) \subseteq \operatorname{Vars}(c)$, a list of input variables,

Out $(c) \subseteq \operatorname{Vars}(c)$, a list of output variables,

and either

Inst $(c)$, a list of instantiations, or

Fct $(c)$, a list of Boolean functions $\operatorname{In}(c) \rightarrow \mathbb{B}$, one function for each output.

Container cells have the Inst attribute while logic cells have the Fct attribute. In the 4-bit adder circuit in Figure 1 there are two cells; the full-adder cell, $f a$, and the 4-bit adder cell, 4bitadder. Vars $(f a)$ is the set $\left\{x_{0}, x_{1}, x_{2}, u_{0}, u_{1}\right\}$. In $(f a)$ is the list $\left[x_{0}, x_{1}, x_{2}\right]$, and $\operatorname{Out}(f a)$ is the list $\left[u_{0}, u_{1}\right]$. The full-adder cell has the Fct attribute; a list with two elements where the first element is the function for the $u_{0}$ output: $x_{0}$ xor $x_{1}$ xor $x_{2}$. The 4bitadder cell has the Inst attribute; a list of four instantiations of $f a$.

Definition 3 (Instantiation) An instantiation $i$ of a cell c has the following attributes:

cell $(i)$, the cell $c$ of which $i$ is an instantiation, $\operatorname{par}(i)$, the cell in which $i$ is located $(i \in \operatorname{Inst}(\operatorname{par}(i)))$, in $(i) \subseteq \operatorname{Vars}($ par $(i))$, a list of input variables, out $(i) \subseteq \operatorname{Vars}($ par $(i))$, a list of output variables.

The instantiation $i$ must further fullfil the requirements: $|\operatorname{in}(i)|=|\operatorname{In}(\operatorname{cell}(i))|$ and $|\operatorname{out}(i)|=\mid$ Out $(\operatorname{cell}(i) \mid$. 
The topmost instantiation of a full-adder cell in the 4bit adder example has cell $(i)=f a, p a r(i)=4$ bitadder, in $(i)=\left[s_{1}, s_{2}, s_{0}\right]$, and out $(i)=\left[s_{9}, s_{10}\right]$.

The outputs of a hierarchical combinational circuit are determined by the inputs. In the case of the 4-bit adder, the outputs are the sum of two 4-bit numbers on the inputs. We use a relation $\operatorname{Rel}(c)$ to capture this relation between the inputs and the outputs of a cell $c$. For a logic cell, Rel is determined by the logic of the gates (the Fct attribute):

$$
\operatorname{Rel}(c)=\bigwedge_{k=1, \ldots, \mid \text { Out }(c) \mid}\left(\operatorname{Out}(c)_{k} \Leftrightarrow F c t(c)_{k}\right) .
$$

(We use characteristic functions to represent relations.) The subscript $k$ indicates the $k$ th element in a list. For example, $\operatorname{Rel}(f a)$ is the relation:

$$
\begin{aligned}
\left(u_{0} \Leftrightarrow x_{0} \oplus x_{1} \oplus x_{2}\right) & \wedge \\
\left(u_{1} \Leftrightarrow\left(x_{0} \wedge x_{1}\right)\right. & \left.\vee\left(x_{2} \wedge\left(x_{0} \vee x_{1}\right)\right)\right) .
\end{aligned}
$$

For container cells, Rel is determined as:

$$
\operatorname{Rel}(c)=\exists v_{\in V} \cdot \bigwedge_{i \in \operatorname{Inst}(c)} \operatorname{Rel}(\operatorname{cell}(i))[\mathrm{Map}],
$$

where $V$ is the set variables which are neither inputs nor outputs, i.e., $V=\operatorname{Vars}(c) \backslash(\operatorname{In}(c) \cup O u t(c))$, and $[M a p]$ is a renaming of $\operatorname{In}(\operatorname{cell}(i))$ and $O u t(\operatorname{cell}(i))$ variables to in $(i)$ and out $(i)$ variables, respectively. The notation $\exists v_{\in V}$ for $V=\left\{v_{1}, v_{2}, \ldots, v_{k}\right\}$ is shorthand for $\exists v_{1} . \exists v_{2}, \cdots . \exists v_{k}$.

For an $\mathrm{HCC}(C, c)$, the relation over the primary inputs and the primary outputs is $\operatorname{Rel}(\mathrm{c})$.

We now define a path and a cut in a cell.

Definition 4 (Path) For a container cell $c$, $a$ path $p=$ $\left\langle p_{1}, \ldots, p_{n}\right\rangle$ is a sequence of variables from $\operatorname{Vars}(c)$ such that for all $k, 1 \leq k<n$, there exists an instantiation $i \in \operatorname{Inst}(c)$, such that $p_{k} \in \operatorname{in}(i)$ and $p_{k+1} \in \operatorname{out}(i)$.

Definition 5 (Cut) $A$ cut $K$ in a container cell $c$ is a set of variables from Vars $(c)$ such that any path $p=$ $\left\langle p_{1}, \ldots, p_{n}\right\rangle$ through $c$ with $p_{1} \in \operatorname{In}(c)$ and $p_{n} \in$ Out $(c)$ contains exactly one variable from the cut $K$.

For both logic and container cells, the input cut is the set In $(i)$ and the output cut is the set Out $(i)$.

The set $\left\{s_{3}, s_{4}, \ldots, s_{10}\right\}$ is a cut in the 4 bitadder container cell. For two cuts in different HCCs we define a cut-relation $H$ as a relation over the values of the variables in the cuts.

Definition 6 (Cut-relation) $A$ cut-relation $H$ between two cuts $K_{1}$ and $K_{2}$ in two cells is a relation over the values of the variables in $K_{1}$ and $K_{2}$, i.e., $H \subseteq \mathbb{B}^{K_{1} \cup K_{2}}$.

A cut-relation over the input cuts of the two circuits in Figure 2 could be:

$$
\bigwedge_{i=0,1,2,5,6}\left(s_{i} \Leftrightarrow t_{i}\right) \wedge \bigwedge_{i=3,4,7,8}\left(s_{i} \Leftrightarrow \neg t_{i}\right),
$$

stating that $s_{i}$ and $t_{i}$ have identical values for $i=$ $0,1,2,5,6$, and that $s_{i}$ and $t_{i}$ have opposite values for $i=3,4,7,8$.

We call a cut-relation between input cuts in two cells for an input relation. Likewise, we call a cut-relation between output cuts for an output relation.

Given a cut-relation $H$ for two cuts $K_{1}$ and $K_{2}$, and two instantiations $i_{1}$ and $i_{2}$ such that the input variables of $i_{1}$ and $i_{2}$ are subsets of $K_{1}$ and $K_{2}$, respectively, we can determine a relation $R_{i n}$ between the input variables of $i_{1}$ and $i_{2}$ :

$$
R_{i n}=\left(\exists v_{\in\left(K_{1} \cup K_{2}\right) \backslash\left(i n\left(i_{1}\right) \cup i n\left(i_{2}\right)\right)}, H\right)[M a p],
$$

where $[M a p]$ is a renaming of $i n\left(i_{1}\right), \operatorname{in}\left(i_{2}\right)$, out $\left(i_{1}\right)$, and $\operatorname{out}\left(i_{2}\right)$ variables to $\operatorname{In}\left(\operatorname{cell}\left(i_{1}\right)\right)$, In $\left(\operatorname{cell}\left(i_{2}\right)\right)$, Out $\left(\operatorname{cell}\left(i_{1}\right)\right)$, and $\operatorname{Out}\left(\operatorname{cell}\left(i_{2}\right)\right)$ variables, respectively.

\section{Cut Propagation}

Given two hierarchical combinational circuits $\mathrm{HCC}_{1}$ $\left(C_{1}, c_{1}\right)$ and $\mathrm{HCC}_{2}\left(C_{2}, c_{2}\right)$, and an input relation $H_{i n}$, the verification problem we consider is to determine whether the outputs satisfy a desired relation $H_{\text {out }}$. Typically, $H_{i n}$ and $H_{\text {out }}$ would represent "the circuits have identical inputs and outputs."

The verification algorithm works by propagating a cut-relation from the inputs to the outputs. Let $H_{0}$ be the input relation $H_{i n}$, a cut-relation between the input cuts of $c_{1}$ and $c_{2}$. We move their cut-relation past instantiations of cells in $c_{1}$ and $c_{2}$ (assuming that $c_{1}$ and $c_{2}$ are container cells). In each step we calculate a new cutrelation, $H_{k+1}$, based on the previous one, $H_{k}$. When the cut-relation has reached the outputs, the resulting cut-relation, $H_{n}$, relates the outputs of $c_{1}$ to the outputs of $c_{2}$. If $H_{n}$ is a subset of $H_{\text {out }}, H_{n} \subseteq H_{\text {out }}$, the circuits have the desired output relation.

\subsection{Example}

Before describing the algorithm in detail, we give an example to illustrate the basic ideas. Consider again the two different implementations of 4-bit adders in Figure 2. The 4-bit adders are described using $s$ and $t$ variables, respectively. The full-adders in the top circuit are described using $x$ (input) and $u$ (output) variables, while the full-adders in the bottom circuit use $y$ and $v$ variables. The $H$ 's represent the cut-relations and the vertical lines indicate the cuts.

Assume $H_{0}$ in Figure 2 is given by (1). We decide to move the cuts from the inputs to the outputs one fulladder at a time and to move the cuts in the two circuits simultaneously. First we calculate the input relation $R_{i n, 1}$ between the leftmost full-adder cell in each of the two circuits using (2):

$$
R_{i n, 1}=\left(x_{0} \Leftrightarrow y_{0}\right) \wedge\left(x_{1} \Leftrightarrow y_{1}\right) \wedge\left(x_{2} \Leftrightarrow y_{2}\right) .
$$


Notice the use of cell variables $x$ and $y$ and not instantiation variables $s$ and $t$, which is important in order to recognize this situation in the future.

Given $R_{i n, 1}$ and the input/output relation $R e l$ for the two full-adders, we can determine the relation between the outputs (we will show later how to do this):

$$
R_{o u t, 1}=\left(u_{0} \Leftrightarrow \neg v_{0}\right) \wedge\left(u_{1} \Leftrightarrow \neg v_{1}\right) .
$$

We move the cuts and determine the new cut-relation $H_{1}$ based on $R_{o u t, 1}$ (again, we will show later how to do this):

$$
\begin{aligned}
& H_{1}=\bigwedge_{i=5,6}\left(s_{i} \Leftrightarrow t_{i}\right) \wedge \\
& \bigwedge_{i=3,4,7,8,9,10}\left(s_{i} \Leftrightarrow \neg t_{i}\right) .
\end{aligned}
$$

In a similar way we propagate the cuts one step further getting $\mathrm{H}_{2}$ :

$$
H_{2}=\bigwedge_{i=5,6,11,12}\left(s_{i} \Leftrightarrow t_{i}\right) \wedge \bigwedge_{i=7,8,9}\left(s_{i} \Leftrightarrow \neg t_{i}\right) .
$$

In the third step, we start by finding the input relation $R_{i n, 3}$ :

$$
R_{i n, 3}=\left(x_{0} \Leftrightarrow y_{0}\right) \wedge\left(x_{1} \Leftrightarrow y_{1}\right) \wedge\left(x_{2} \Leftrightarrow y_{2}\right) .
$$

This is identical to the relation $R_{i n, 1}$ from the first step. The full-adders in the third step are also identical to the full-adders in the first step, and thus we can immediately reuse the output relation $R_{\text {out }, 1}$ instead of calculating $R_{o u t, 3}$. We update the $H_{2}$ relation using $R_{o u t, 1}$ and obtain $\mathrm{H}_{3}$ :

$$
\begin{aligned}
H_{3}= & \left(s_{9} \Leftrightarrow \neg t_{9}\right) \wedge\left(s_{11} \Leftrightarrow t_{11}\right) \wedge \\
& \left(s_{13} \Leftrightarrow \neg t_{13}\right) \wedge\left(s_{14} \Leftrightarrow \neg t_{14}\right) .
\end{aligned}
$$

Similarly in the fourth step the input relation is found to be identical to that of the second step and the full-adders in the second and the fourth step are identical. We update the relation $\mathrm{H}_{3}$, and get the final output relation $\mathrm{H}_{4}$;

$$
\begin{gathered}
H_{4}=\left(s_{9} \Leftrightarrow \neg t_{9}\right) \wedge\left(s_{11} \Leftrightarrow t_{11}\right) \wedge\left(s_{13} \Leftrightarrow \neg t_{13}\right) \\
\\
\wedge\left(s_{15} \Leftrightarrow t_{15}\right) \wedge\left(s_{16} \Leftrightarrow t_{16}\right) .
\end{gathered}
$$

We observe that the sum-bits of the first and third pair of adders have opposite values while the sum-bits of the second and fourth pair of adders are pairwise equivalent.

\subsection{Moving Cuts}

We distínguish between two ways of moving cuts: Build and Propagate, Build determines the input/output relation Rel for a cell $c$ and uses it to calculate the new cut-relation by moving the cut past an instantiation of cell $c$. Propagate moves cuts past two cells simultaneous by calculating the input relation $R_{\text {in }}$ between the inputs of the two cells and from that calculate the output relation $R_{\text {out }}$ for the same pair of cells. In the example above we only used Propagate.
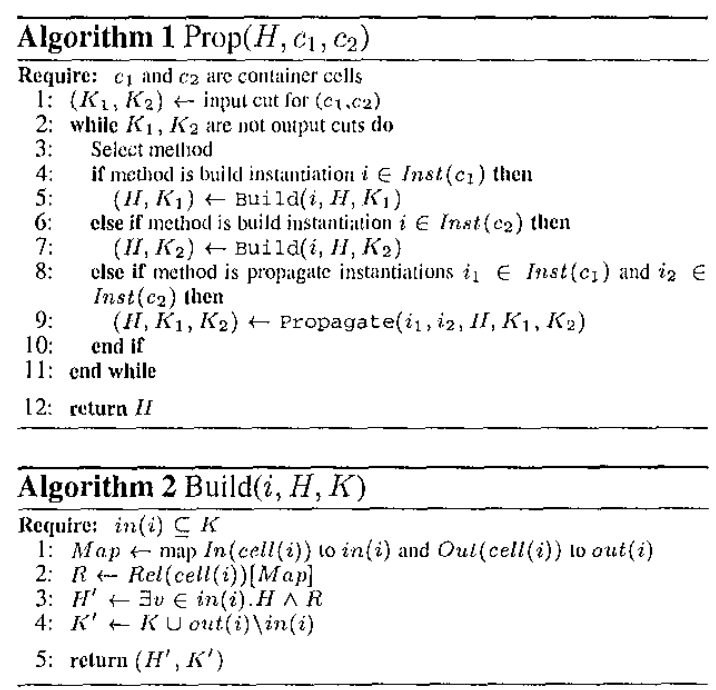

Algorithm $\$ shows the pseudo-code for the overall algorithm Prop. The algorithm moves a cut through two container cells by for each step selecting either the Build or the Propagate algorithm. In the example, $\operatorname{Prop}\left(H_{0}, 4\right.$ bitadder $_{1}, 4$ bitadder $\left._{2}\right)$ calculates the output relation $\mathrm{H}_{4}$, where 4 bitadder ${ }_{1}$ and 4 bitadder 2 are the two different descriptions of 4-bit adders.

Algorithm 2 shows the pseudo-code for Build. It takes three inputs: an instantiation $i$, a cut-relation $H$, and a cut $K$. It is assumed that all input variables for $i$ are in the cut. The lines 1 and 2 calculate the input/output relation for $\operatorname{cell}(i)$ using instantiation variables, line 3 calculates the new cut-relation, and line 4 calculates the new cut.

The Propagate algorithm shown in Algorithm 3 considers two cell instantiations at a time; one in each circuit. Propagate takes five arguments; two instantiations $i_{1}$ and $i_{2}$, two cuts $K_{1}$ and $K_{2}$, and a cut-relation $H$ over the cuts. The result is a new cut-relation and two new cuts. It is assumed that the input variables of the cell instantiations $i_{1}$ and $i_{2}$ belong to the cuts $K_{1}$ and $K_{2}$, respectively.

In line I Propagate calculates, using (2), the input relation $R_{i n}$ between $i_{1}$ and $i_{2}$ based on the cut-relation

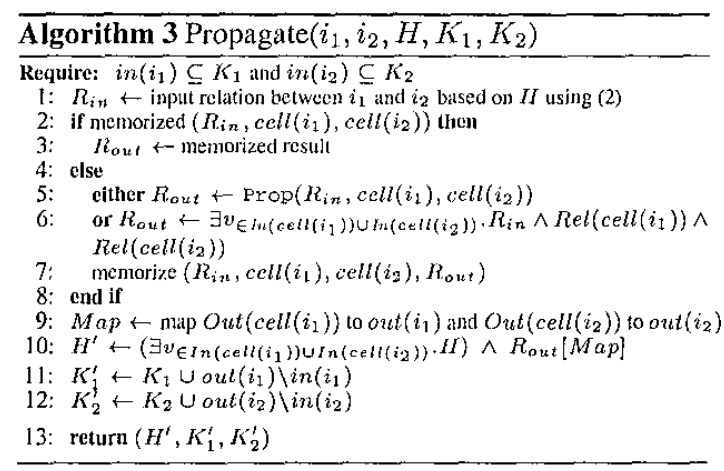


$H$. The input relation $R_{i n}$ is described in cell variables, not in instantiation variables. Next, we calculate the output relation $R_{\text {out }}$ for $i_{1}$ and $i_{2}$. If we have previously propagated a similar cut past the same cells, we reuse the previous result (line 3 ). Otherwise we have two ways of calculating $R_{\text {out }}$. If both $i_{1}$ and $i_{2}$ are instantiations of container cells, we can propagate the cut through these instantiations (line 5) by calling Prop, which allows us to use Propagate on the container cells. Alternatively, we compute $R_{\text {out }}$ from the input/output relation $R e l$ for each of the instantiations $i_{1}$ and $i_{2}$ (line 6). This resembles calling Build twice. The rest of the algorithm updates the cuts and calculates the new cut-relation.

In the example, we calculated (4) in line 6 and we calculated the updated cut-relation $H_{1}(5)$ in line 10 .

\subsection{Build vs. Propagate}

Build works by constructing a representation of the input/output relation for a cell which is used to update the cut-relation $H$. Such a relation captures the functionality of the cell. Using Build on the top cell corresponds to the standard verification method of building the OBDD for the entire circuit. While this works well for smaller circuits, the OBDDs tend to become quite large for more complex circuits.

Propagate works by moving a relation between input variables of two cells to a relation between output variables of the same two cells. In case of container cells, Propagate moves the cuts one step at a time past instantiations of cells in the container cells. It avoids constructing an OBDD for the functionality of a cell as long as possible. For logic cells it is necessary to construct such an OBDD. However, this OBDD represents only the functionality of a part of the circuit, not the whole circuit, and it is therefore more manageable.

The use of Propagate may cause loss of information since it requires contruction of the input relation $R_{\text {in }}$ between the cell inputs. Consider the two equivalent circuits in Figure 3 . The input cut for the top circuit is $K_{1}=\left\{s_{0}, s_{1}\right\}$ and for the bottom circuit it is $K_{2}=\left\{t_{0}, t_{1}\right\}$. Let $H_{0}$ be the cut-relation $\left(s_{0} \Leftrightarrow t_{0}\right) \wedge\left(s_{1} \Leftrightarrow t_{1}\right)$ when calling Propagate. We want to move the cuts past the negation cells. The new cuts contain the variables $s_{1}$ and $s_{2}$, and $t_{0}$ and $t_{2}$. We

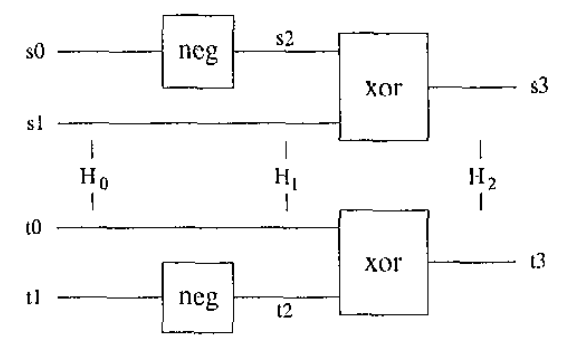

ligure 3: Two combinational circuits. The relation $H_{0}$ between the wires in the input cuts is $\left(s_{0} \Leftrightarrow t_{0}\right) \wedge\left(s_{1} \Leftrightarrow t_{1}\right)$. build the input relation $R_{i n}$ for the two negation cells:

$$
\begin{aligned}
R_{i n} & =\left(\exists v_{\in\left(K_{1} \cup K_{2}\right) \backslash\left(\operatorname{in}\left(i_{1}\right) \cup i n\left(i_{2}\right)\right)} \cdot H_{0}\right)[\text { Map }] \\
& =\left(\exists v_{\in\left\{s_{1}, t_{0}\right\}} \cdot\left(s_{0} \Leftrightarrow t_{0}\right) \wedge\left(s_{1} \Leftrightarrow t_{1}\right)\right)[M a p] \\
& =t r u e,
\end{aligned}
$$

where $i_{1}$ and $i_{2}$ are instantiations of the two negation cells. In this case $R_{\text {in }}$ evaluates to true, meaning that the inputs are unrelated; knowing the value of $s_{0}$ does not imply a particular calue of $t_{1}$, i.e., they are unrelated. $R_{\text {in }}=$ true results in $H_{1}$ also being true and not the expected $\left(s_{1} \Leftrightarrow \neg t_{2}\right) \wedge\left(\neg s_{2} \Leftrightarrow t_{0}\right)$.

The problem is that $H_{0}$ does not relate the cut variables $s_{0}$ and $t_{1}$. In general we can state that Propagate $\left(i_{1}, i_{2}, H, K_{1}, K_{2}\right)$ works without loss of information if the cut-relation $H$ can be split in two parts: one part containing the variables in $M=i n\left(i_{1}\right) \cup i n\left(i_{2}\right)$ and one part containing the remaining variables:

$$
H \Longleftrightarrow\left(\exists v_{\in M} \cdot H\right) \wedge\left(\exists v_{\in\left(K_{1} \cup K_{2}\right) \backslash M} \cdot H\right)
$$

If $H$ can be written as in (6), Propagate determines the exact cut-relation $H^{\prime}$. Otherwise, it gives a conservative approximation to the output relation $H_{c x a c i} \subseteq$ $H_{\text {approx }}$.

\section{EXPERIMENTAL RESULTS}

To test the proposed method, we have implemented it using OBDDs to represent the characteristic functions of relations. The canonicity allows us to recognize memorized results (line 2 in algorithm 3 ) in constant time. We have built hierarchical adder and multiplier circuits of different sizes. Each $n$-bit adder consists of two $n / 2$ bit adders. We built one series of adders using the fulladder cells from Figure 1, and another series of adders using two different types of full-adder cells: one fulladder outputting a negated carry-out, and one receiving a negated carry-in signal. The verification task is to verify that given identical inputs, the adders from the two series have identical outputs. The left part of Table 1 shows the runtimes for this experiment. The strategy for moving cuts was to use Propagate whenever $H$ can be written as in (6), otherwise we use Build. Becatse of the reuse of previously calculated results, we only apply Propagate a number of times proportional to $\log _{2}(n)$ for $n$-bit adders.

Using standard OBDD techniques, it is possible to get results comparable to those in Table 1 for the verification of adders since the addition function has a small OBDD representation (when using an appropriate variable order). However, OBDDs are very sensitive to the chosen variable ordering, and using a bad variable order results in OBDDs of size exponential in $n$ making it infeasible to build the OBDDs for the adders. Our proposed method is not sensitive to the variable ordering of the adders as we never build OBDDs representating the functionality of the circuits. 
Table 1: Runtimes in seconds on a $500 \mathrm{MHz}$ Digital Alpha to verify pairs of hierarchical adders (left) and pairs of hicrarchical multipliers (right) against each other.

\begin{tabular}{rr|rr}
\hline $\begin{array}{r}\text { Adder } \\
\text { [bits] }\end{array}$ & $\begin{array}{r}\text { Runtime } \\
\text { [sec] }\end{array}$ & $\begin{array}{r}\text { Multiplicr } \\
\text { [bits] }\end{array}$ & $\begin{array}{r}\text { Runtime } \\
\text { [sec] }\end{array}$ \\
\hline 64 & 0.2 & 32 & 2.9 \\
128 & 0.3 & 64 & 14.6 \\
256 & 0.5 & 128 & 87.4 \\
512 & 0.8 & 256 & 649 \\
1024 & 1.6 & & \\
\hline
\end{tabular}

We tested the sensitivity to errors of the cutpropagation method by introducing errors into the adders by switching wires around close to the leaves and close to the root in the hierarchy - errors typically arising if wrong parameter lists are given in the circuit descriptions. None of the modifications cause the runtimes to increase significantly.

While adders are easy to handle using OBDDs, multipliers are notoriously difficult. We construct multipliers as series of adders and shifters. From the two different types of adders in the previous experiment, we create two different types of multipliers. The verification task is to verify the pairwise equivalence of outputs given the pairwise equivalence of inputs. One complication is that the outputs of a multiplier are not unrelated. For example, it is not possible for all outputs to be 1 simultaneously ${ }^{1}$. When calculating the cut-relations, such restrictions are included in the relations. This means that the cut-relations contain more information than we need. Repeated use of Propagate, even when the cutrelation cannot be written as 6 and thus Propagate causes loss of information, turns out to be exactly what is needed to "forget" this extra information. The right part of Table 1 shows the results from running the multiplier experiments.

\section{Conclusion}

We have presented a method based on cut-propagation for obtaining a relation between the outputs of two hierarchically specified combinational circuits. The key new feature of the method is it ability to exploit the hierarchy in the circuit description to reuse previously calculated results in the verification. We have demonstrated the power of the method by verifying large adders and multipliers.

\section{ACKNOWLEDGEMENTS}

Thanks to Jakob Lichtenberg for suggestions to (6).

\footnotetext{
'For an $n$-bit multiplier, the grealest result is $\left(2^{n}-1\right)^{2}$, which is less than $2^{2 n}-1$, where $2^{2 n}-1$ corresponds to 1 on all outputs.
}

\section{REFERENCES}

[1] H. R. Andersen and H. Hulgaard. Boolcan expression diagrams. In IEEE Symposium on Logic in Computer Science (LICS), July 1997.

12] D. Brand. Verification of large synthesized designs. In Proc. International Conf. Compater-Aided Design (ICCAD), pages 534-537, 1993.

[3] R. E. Bryant. Graph-based algorithms for Boolean function manipulation. IEEE Transactions on Computers, 35(8):677-69 I, Aug. 1986.

[4] R. E. Bryant and Y.-A. Chen. Verification of arithmetic functions with binary moment diagrams. In Proc. ACM/IEEE Design Automation Conference (DAC), pages 535-541, 1995.

[5] E. Cerny and C. Mauras. Tautology checking using crosscontrollability and cross-observability relations. In Proc. International Conf. Computer-Aided Design (ICCAD), 1990.

[6] M. Fujita. Verification of arithmetic circuits by comparing two similar circuits. In Computer Aided Verification (CAV), Lecture Notes in Computer Science, pages 159168. Springer-Vcrlag, 1996.

[7] 1. Gergov and C. Mcinel. Efficient Boolcan manipulation with OBDD's can be extended to FBDD's. ILEE Transactions on Computers, 43(10):1197-1209, Oct. 1994.

[8] H. Hulgaard, P. F. Williams, and H. R. Andersen. Equivalence checking of combinational circuits using Boolean expression diagrams. IEEE Transactions on Computer Aided Design, July 1999.

[9] J. Jain, J. Bitner, M. S. Abadir, J. A. Abraham, and D. S. Fussell. Indexed BDDs: Algorithmic advances in techniques to represent and verify Boolean functions. IEEE Transactions on Computers, 46(11):1230-1245, Nov. 1997

[10] J. Jain, R. Mukherjee, and M. Fujita. Advanced verification techniques based on lcarning. In Proc. $A C M / I E E E$ Design Altomation Conference (DAC), pages 629-634, 1995.

[11] U. Kebschull, E. Schubert, and W. Rosenstiel. Multilevel logic synthesis based on functional decision diagrams, In Proc. European Conference on Design Automation (EDAC), pages 43-47, 1992.

[12] A. Kuchlmann and F. Krohm. Equivalence checking using cuts and heaps. In Proc. ACM/IELE Design Automation Conference (DAC), volume 34, pages 263-268, 1997.

[13] W. Kunz and D. K. Pradhan. Recursive learning: A new implication technique for efficient solutions to CAD problems - test, verification, and optimization. IEEE Transactions on Computer Aided Design, 13(9):11431158, Sept. 1994.

[14] Y. Matsunaga. An efficient equivalence checker for combinational circuits. In Proc. ACM/IEEE Design Automation Conference (DAC), pages 629-634, 1996.

[15] D. K. Pradhan, D. Paul, and M. Challerjee. VERILAT: Verification using logic augmentation and transformations. In Proc. International Conf. Computer-Aided De. sign (ICCAD), Nov. 1996.

[16] C. van Eijk. Sequential equivalence checking without state space traversal. In Proc. International Conf. on Design Automation and Test of Electronic-based Systems (DATE), 1998

[17] C. van Eijk and G. L. J. M. Janssen. Exploiting structural similarities in a BDD-based verification method. In Theorem Provers in Circuit Design, number 901 in Lecture Notes in Computer Science, pages 110-125. SpringerVerlag, 1994 